\title{
Correction to: Efficacy of Ronopterin (VAS203) in Patients with Moderate and Severe Traumatic Brain Injury (NOSTRA phase III trial): study protocol of a confirmatory, placebocontrolled, randomised, double blind, multi-centre study
}

Frank Tegtmeier ${ }^{1}$, Reinhard Schinzel ${ }^{1 *}$, Ronny Beer ${ }^{2}$, Diederik Bulters ${ }^{3}$, Jean-Yves LeFrant ${ }^{4}$, Joan Sahuquillo ${ }^{5}$, Andreas Unterberg ${ }^{6}$, Peter Andrews ${ }^{7}$, Antonio Belli ${ }^{8}$, Javier Ibanez ${ }^{9}$, Alfonso Lagares ${ }^{10}$, Michael Mokry ${ }^{11}$, Harald Willschke ${ }^{12}$, Charlotte Flüh ${ }^{13}$, Erich Schmutzhard ${ }^{2}$ and on behalf of the NOSTRA Investigators

Correction to: Trials (2020) 21:80

https://doi.org/10.1186/s13063-019-3965-4

After publication of our article [1] the authors have notified us that one of the names has been incorrectly spelled.

- Original name spelling: Charlotte Flühe

- Correct name spelling: Charlotte Flüh

The original article has been corrected.
Published online: 12 February 2020

\section{Reference}

1. Tegtmeier, et al. Efficacy of Ronopterin (VAS203) in Patients with Moderate and Severe Traumatic Brain Injury (NOSTRA phase III trial): study protocol of a confirmatory, placebocontrolled, randomised, double blind, multi-centre study. Trials. 2020;21:80. https://doi.org/10.1186/s13063-019-3965-4.

\footnotetext{
Author details

${ }^{1}$ vasopharm GmbH, Würzburg, Germany. ${ }^{2}$ Medizinische Universität Innsbruck, Innsbruck, Austria. ${ }^{3}$ Wessex Neurological Centre University Hospital, Southampton, UK. ${ }^{4}$ Hopital Universitaire Caremeau, Nimes, France. ${ }^{5}$ Vall d'Hebron University Hospital, Barcelona, Spain. ${ }^{6}$ Universitätsklinikum Heidelberg, Heidelberg, Germany. Western General Hospital Lothian University, Edinburgh, UK. ${ }^{8}$ Queen Elizabeth Hospital, Birmingham, UK. ${ }^{9}$ Espases University Hospital, Palma de Mallorca, Spain. ${ }^{10}$ Hospital Universitario 12 de Octubre, Madrid, Spain. ${ }^{11}$ LKH - Universitätsklinikum Graz, Graz, Austria. ${ }^{2}$ Medizinische Universität Wien, Wien, Austria.

${ }^{13}$ Universitätsklinikum Schleswig-Holstein, Kiel, Germany.
}

* Correspondence: schinzel@vasopharm.de

1 vasopharm GmbH, Würzburg, Germany

Full list of author information is available at the end of the article 